\title{
Optimizing the Motion for Robotic Snap Assembly Using FEM
}

\author{
Peihao Shi, Kensuke Harada, Weiwei Wan, Ixchel G. Ramirez \\ Department of Systems Innovation, Osaka University, 1-3 Machikaneyama-cho \\ Toyonaka, Osaka, Japan. \\ Juan Rojas \\ School of Mechanical and Electrical Engineering, GDUT, 100 Waihuanxi Lu, Gongxue 2, 105 \\ Guangdong University of Technology, Guanzhou, Guandong, China \\ Hiromu Onda \\ AIST, 1-1-1Umezono \\ Tsukuba, Ibaraki, Japan
}

\begin{abstract}
In this paper, we aim to provide a better assembly method for a snap joints assembly task. We create a 3D cellphone model and use ADAMS simulation environment to analyze the relative motion between screen and backer part. We focus on two points in this research. 1) Two kinds of relative motion between the screen and backer parts, i.e., the rotation-based and the translation-based methods, are compared, and 2) difference between assembly and disassembly is analyzed. By using the maximum elastic energy in an assembly process, we show that 1) the rotation-based assembly motion has better robustness than the translation-based assembly motion in cellphone assembly tasks when we set the same initial position error. 2) The rotation-based assembly method is more effective when we disassembly the cellphone screen part.
\end{abstract}

Keywords: Robotic Assembly, Snap joints, Strain Energy.

\section{Introduction}

Snap joints connection is one of the typical examples of plastic parts assemblage. As the widely application and mass-production of plastic parts, snap joints assembly is becoming more and more popular in assemble method of Industrial products. In snap joint assembly, the connection between two parts is built by elastic deformation of snap joints. [1] Fig.1 shows an example of the snap assembly task. Because of the elastic deformation of the snap joints, it becomes relatively hard for an industry robot to finish snap joints assembly task [2-3]. As for the research on robotic snap joints assembly.
Rojas et al. have proposed a force control method [4-6] and identification of assembly state for the snap assembly problem.

Rojas et al. focus on the identification of assembly state in 2012 and prove that the rotation-based assembly method is more robust against the error of part's pose than the translation-based method in robotic snap assembly [7].

In 2016, we proposed the Extend Directional Blocking graph for snap assembly [7]. We also proposed the Maximum Elastic Energy (MEE) used for the 
Extended Directional Blocking graph to evaluate the easiness or difficulty of snap assembly where smaller MEE implies the easiness of snap joints assembly [8].We analyzed the motion of snap joints assembly based dynamic simulation by using FEM. We proposed a simulation based method to compute the MEE [9-10].

In this paper we aim to provide a better assembly method for a cellphone snap joints assembly task. We create a 3D model of a cellphone, and use ADAMS simulation environment to analyze the assembly process of the cellphone. Using the Maximum Elastic Energy (MEE) as an index for evaluating the quality of a snap joints assembly tasks, we compare two kinds of assembly methods, i.e., rotation-based and translation-based methods. We also compare the difference of MEE in assembly and disassembly when we choose different assembly method.

By using the maximum elastic energy in an assembly process, we show that 1) Rotation-based assembly motion has better robustness than the translation-based assembly motion in cellphone assembly tasks. When we set the same initial position error. 2) The rotation-based assembly method is more effective when we disassembly the cellphone screen part.

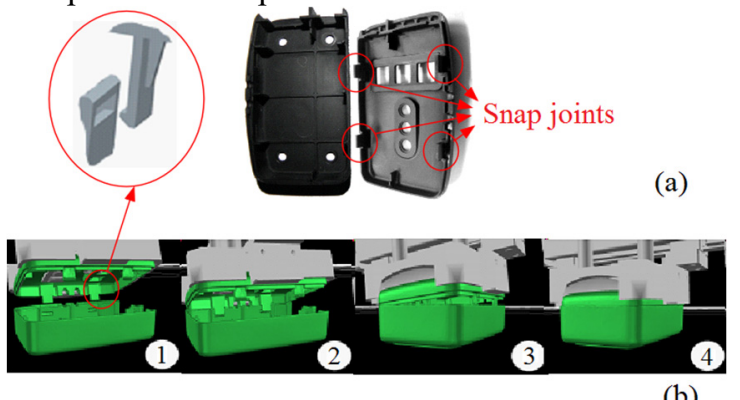

(b)

Fig.1 Snap assembly task

\section{Approach and Definitions}

We create a 3D model of a cellphone used in ADAMS physics simulation environment where the screen part contains four snap joints as shown in Fig. 2. We simulate the assembly process by using the redefined relative motion between two parts. Throughout a physics simulation of a snap joint assembly, we consider obtaining the function curve of strain energy. And select the MEE from the strain energy curve.

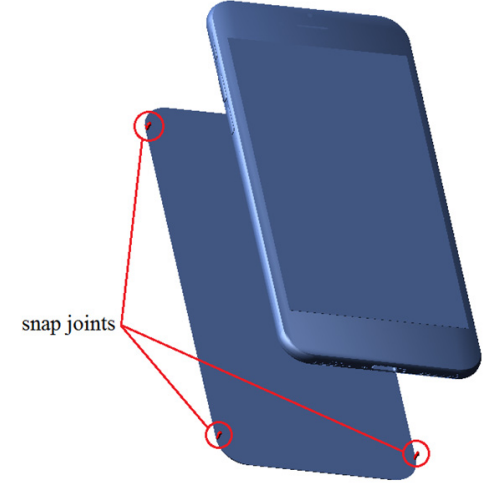

Fig.2 3D cellphone model.

We consider $E i(s)$ as the total elastic energy in the assembly process. Here, $\mathrm{s}$ is a scalar ranging from 0 to 1 . It indicates the states of assembly. 0 is the starting state. 1 is the goal state. A value between them is an intermediate state. The maximum elastic energy (MEE) $U i$ is being defined for snap assembly/disassembly task of Part $i$. [8]

$$
U_{i}=\max _{0 \leq s \leq 1} E_{i}
$$

We use Adams physics simulation environment analyze the assembly process. We can get the strain energy-duration function curve in Adams. And select the MEE from the curve. By compare the MEE of different assembly methods, we can choose the better assembly method which the MEE is smaller.

\section{Experiment and Analysis.}

In this section, we set two kinds of relative motion for cellphone assembly task, the rotation based relative motion is shown in Fig. 3, and translation based relative motion is shown in Fig. 4. We compute and compare the MEE of rotation-based/translation-based relative motion. And the better assembly method is be defined as the one bring smaller MEE in assembly process.

\subsection{Initial Position Errors.}

In robotic assembly tasks. Influence of initial positon errors always been used as a criterion of robustness. In our experiment, we set the initial position errors of screen part to $0 \mathrm{~mm}, 1 \mathrm{~mm}, 2 \mathrm{~mm}$ (Fig. 3 ) to compare the rotationbased and translation-based assembly motion. 


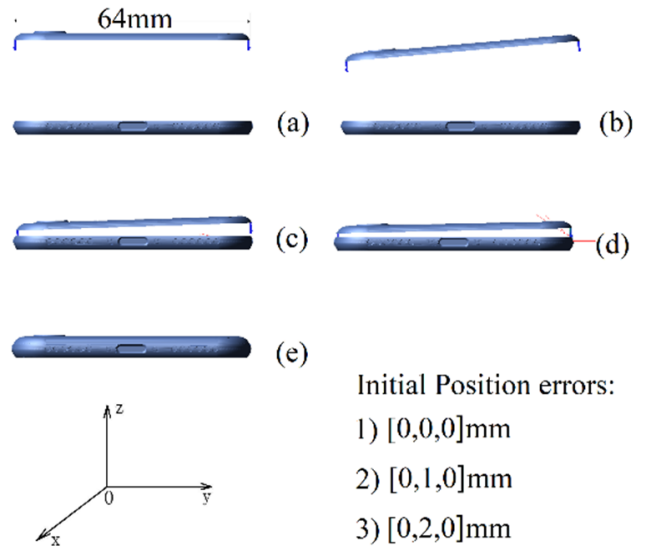

Fig.3 Rotation-based relative motion and Initial position errors.

Fig. 5 and Fig 6 shows the simulation results when we set the initial position errors to $0 \mathrm{~mm}$. The result shown the strain energy of translation based-motion $(1.19 \mathrm{~J})$ is bigger than the rotation-based motion $(0.94 \mathrm{~J})$.

Fig. 7 and Fig. 8 shows the simulation result when we set the initial position errors to $1 \mathrm{~mm}$. The result shown the strain energy of translation based-motion $(2.17 \mathrm{~J})$ is bigger than the rotation-based motion (1.12J). Fig. 9 and Fig. 10 shows the simulation result when we set the initial position errors to $1 \mathrm{~mm}$. The result shown the strain energy of translation based-motion $(3.51 \mathrm{~J})$ is bigger than the rotation-based motion (1.13J).

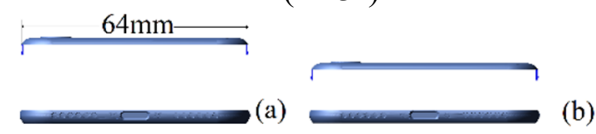

(c)

Fig.4 Translation-based relative motion.

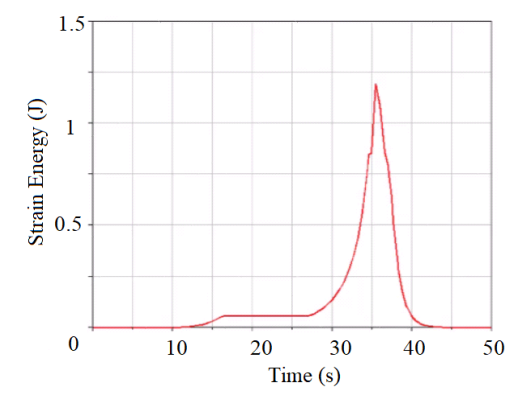

Fig. 5 Strain energy of $0 \mathrm{~mm}$ position error in translation-based assembly

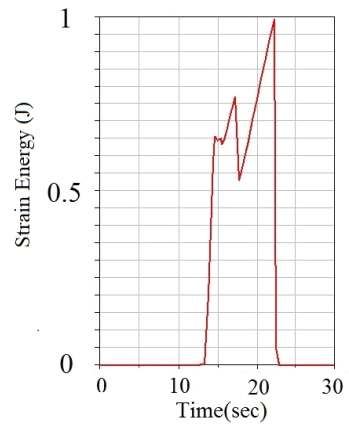

Fig. 6 Strain energy of $0 \mathrm{~mm}$ position error in rotation-based assembly

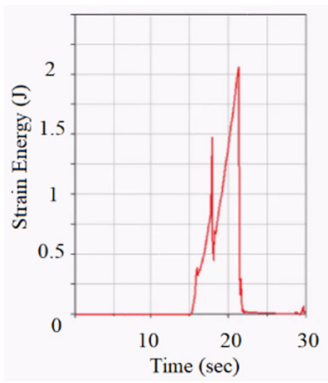

Fig. 7 Strain energy of $1 \mathrm{~mm}$ position error in translation-based assembly

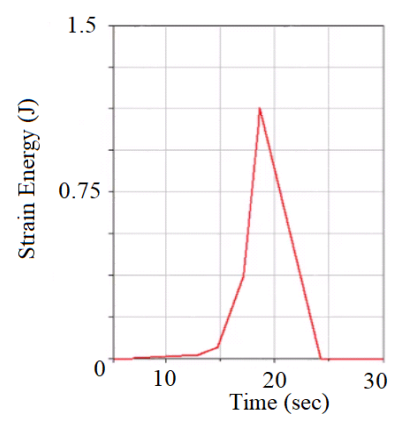

Fig. 8 Strain energy of $1 \mathrm{~mm}$ position error in rotation-based assembly

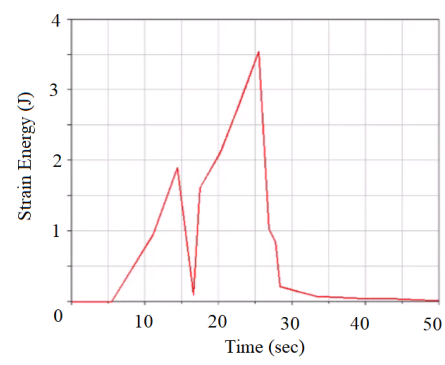

Fig. 9 Strain energy of $2 \mathrm{~mm}$ position error in translation-based assembly 


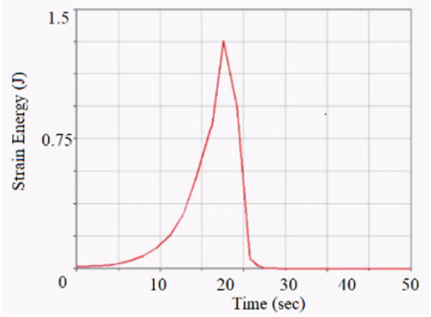

Fig. 10 Strain energy of $2 \mathrm{~mm}$ position error in rotation-based assembly

The simulation result shown a conclusion: when we set the same initial position errors, the MEE in rotationbased assembly process is smaller than translation-based assembly process. And proved rotation-based assembly method has better robustness than translation-based assembly method.

\section{2 difference of assembly and disassembly.}

As the second point, we analyze the difference of assembly and disassembly in two kind of assembly methods. The MEE is different in assembly and disassembly when we use translation-based assembly method. Fig. 11 shows the MEE in assembly/disassembly process based on translation-based relative motion. The MEE in assembly process is $1.19 \mathrm{~J}$, it is smaller than the MEE in disassembly process (1.52J). Nevertheless, Fig. 12 shows the MEE in assembly/disassembly process when we use rotation-based assembly method, the MEE is almost same in assembly and disassembly process (0.94J).

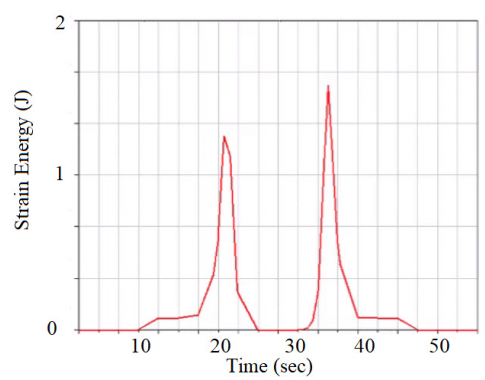

Fig. 11 Strain energy of assembly/disassembly process in translation-based assembly

The simulation result shown that the rotation based assembly method is more effective when we disassembly the cellphone screen part.

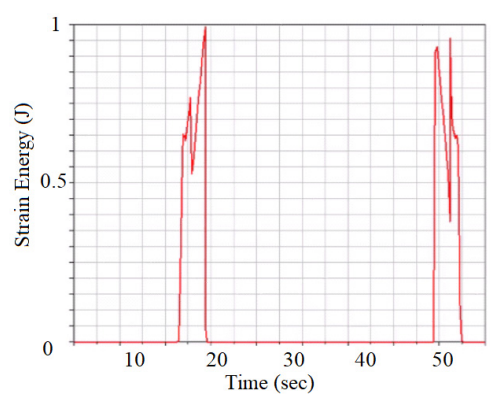

Fig. 12 Strain energy of assembly/disassembly process in rotation-based assembly

\section{Conclusions.}

This paper analyzes the $3 \mathrm{D}$ cellphone snap joints assembly task. We compare two kinds of assembly method from the following points. First is the initial position errors of screen part. Second is difference between assembly and disassembly.

We use ADAMS to simulate the snap joints assembly motion and get the strain energy curve to compute the MEE. Take the cellphone screen snap assembly task for example.

We proposed method evaluates the MEE of rotation based and translation based assembly, selects a better assembly method for a cellphone snap joints assembly task. We also compare the difference of MEE in assembly and disassembly when we choose different assembly method.

Based on the simulation result. We can draw the following conclusions: the rotation-based assembly method has better robustness than translation-based assembly method when we set same initial positon error. The rotation-based assembly method is more effective when we disassembly the cellphone screen part.

\section{References}

1. Randall H. Wilson and Jean-Claude Latombe, Geometric reasoning about mechanical assembly, Artificial Intelligence, 71, 1994, pp.371-396.

2. H. Chang and T.-Y. Li, Assembly Maintainability Study with Motion Planning, Proc. of IEEE Int. Conf. on Robotics and Automation, 1995, pp. 1012-1019.

3. D. Koditscheck, An Approach to Autonomous Robot Assembly, Robotica, vol. 12, no. 2 , 1994, pp. 137-155.

4. J. Rojas, K. Harada et al., A Constraint-Based Motion Control Strategy for Cantilever Snap Assemblies, Proceedings of 2012 IEEE International Conference on Mechatronics and Automation, 2012, pp.1815-1821. 
5. J. Rojas, K. Harada et al., A Relative-Change-Based Hierarchical Taxonomy for Cantilever-Snap Assembly Verification, IEEE/RSJ International Conference on Intelligent Robots and Systems, 2012, pp.356-363.

6. J. Rojas, K. Harada et al., Early Failure Characterization of Cantilever Snap Assemblies using the PA-RCBHT, IEEE International Conference on Robotics \& Automation (ICRA), 2014, pp.3370-3377.

7. J. Rojas, K. Harada et al., Strategies, Controllers, and Coordination: Bi-Manual Snap Assembly Automation, International Conference on Robotics and Biomimetics, 2014, pp.1266-1271.

8. P. Shi, K. Harada et al., Extended Directional Blocking Graph (EDBG) for Snap Joint Assembly. The 34-th Annual Conference of the RSJ, 1B2-08, 2016.9.7, pp64-64.

9. P. Shi, K. Harada et al., Initial Motion Analysis for Pushing Point of Robotic Snap Assembly. The 35-th Annual Conference of the RSJ, 1L3-04, 2017.9.12, pp1919.

10. P. Shi, K. Harada et al., Motion Analysis for Realizing Robotic Snap Assemblies. The Japan Society of Mechanical Engineers, Robotics and Mechatronics Conference, 2P1-A03, 2017.5.12, pp 181-181. 